\title{
Electrophysiological Signature Reveals Laminar Structure of the Porcine Hippocampus
}

(D)Alexandra V. Ulyanova, ${ }^{1 *}$ Paul F. Koch, ${ }^{1 *}$ Carlo Cottone, ${ }^{1}$ DMichael R. Grovola, ${ }^{1,2}$ Christopher D. Adam, ${ }^{1,2}$ Kevin D. Browne, ${ }^{1,2}$ Maura T. Weber, ${ }^{1}$ Robin J. Russo, ${ }^{1}$ Kimberly G. Gagnon, ${ }^{1}$ Douglas $\mathrm{H}$. Smith, ${ }^{1}$ - H. Isaac Chen, ${ }^{1,2}$ Victoria E. Johnson, ${ }^{1}$ D. Kacy Cullen, ${ }^{1,2^{\dagger}}$ and ${ }^{\mathbb{D}}$ John A. Wolf ${ }^{1,2^{\dagger}}$

DOI:http://dx.doi.org/10.1523/ENEURO.0102-18.2018

${ }^{1}$ Department of Neurosurgery, University of Pennsylvania, Philadelphia, PA 19104 and ${ }^{2}$ Corporal Michael J. Crescenz Veterans Affairs Medical Center, Philadelphia, PA 19104

\section{Visual Abstract}
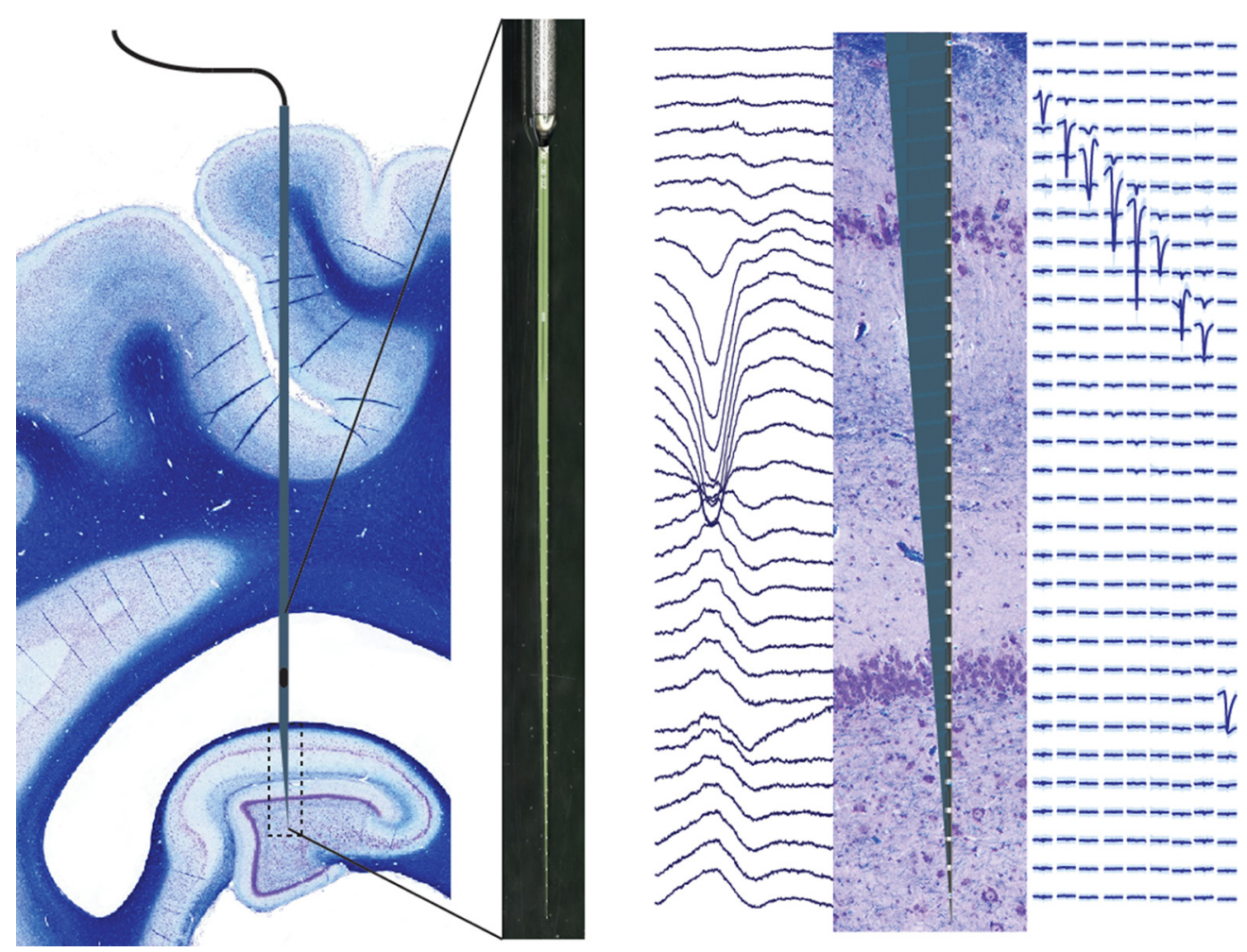

\section{Significance Statement}

The hippocampus is central to working and episodic memory and is critically affected by diverse disease processes. To investigate hippocampal electrophysiology in translational large animal models, we developed an imaging-free stereotaxis and intraoperative electrophysiology methodology with custom silicon probes to precisely localize probe placement within the hippocampal laminar structure. We report for the first time the profile of single units and local field potentials in the pig dorsal hippocampus and relate them to a histologic description. This characterization forms the basis for accessible translational pig models to study diseases of the central nervous system affecting hippocampal circuitry in the large animal gyrencephalic brain, as well as the groundwork for potential awake behaving neurophysiology of the porcine hippocampus. 
The hippocampus is integral to working and episodic memory and is a central region of interest in diseases affecting these processes. Pig models are widely used in translational research and may provide an excellent bridge between rodents and nonhuman primates for CNS disease models because of their gyrencephalic neuroanatomy and significant white matter composition. However, the laminar structure of the pig hippocampus has not been well characterized. Therefore, we histologically characterized the dorsal hippocampus of Yucatan miniature pigs and quantified the cytoarchitecture of the hippocampal layers. We then utilized stereotaxis combined with single-unit electrophysiological mapping to precisely place multichannel laminar silicon probes into the dorsal hippocampus without the need for image guidance. We used in vivo electrophysiological recordings of simultaneous laminar field potentials and single-unit activity in multiple layers of the dorsal hippocampus to physiologically identify and quantify these layers under anesthesia. Consistent with previous reports, we found the porcine hippocampus to have the expected archicortical laminar structure, with some anatomical and histological features comparable to the rodent and others to the primate hippocampus. Importantly, we found these distinct features to be reflected in the laminar electrophysiology. This characterization, as well as our electrophysiology-based methodology targeting the porcine hippocampal lamina combined with high-channel-count silicon probes, will allow for analysis of spike-field interactions during normal and disease states in both anesthetized and future awake behaving neurophysiology in this large animal.

Key words: Custom silicon probes; hippocampal cytoarchitecture; in vivo electrophysiology; pig

\section{Introduction}

The foundations of experimental neuroscience are largely rodent and nonhuman primate neuroanatomy, neurophysiology, and neurochemistry. Rodents and nonhuman primates have also been the dominant species used to study neurologic deficits involving the hippocampus, such as memory disruption following traumatic brain injury (TBI), neurodegeneration, and epilepsy (Levin, 2003; Buzsáki, 2015; Jang and Chung, 2016). While the use of nonhuman primates reduces problems due to species differences, these models are somewhat limited by economic and ethical restraints (Goodman and Check, 2002; Vink, 2018). Since gyrencephalic brain structure and appropriate gray/white matter ratios may be important for accurate modeling of CNS disorders, pigs have recently been proposed as an additional model for translational neuroscience research (Lind et al., 2007).

Much of the basic neuroanatomical characterization of the porcine brain and the hippocampus in particular has

Received March 13, 2018; accepted September 4, 2018; First published September 17, 2018.

The authors declare no competing financial interests.

Author contributions: AVU, PFK, and JAW designed research; AVU, PFK, CDA, MRG, KDB, MTW, RJR, KGG, HIC, VEJ, and JAW performed research; DHS contributed unpublished reagents/analytical tools; AVU, CC, CDA, and PFK analyzed data; AVU, PFK, DKC, and JAW wrote the paper.

Funding sources: The Department of Veterans Affairs, IK2-RX001479, I01RX001097. The National Institutes of Health, NINDS R01-NS-101108-01, T32NS043126. CURE Foundation, Taking Flight Award. DoD ERP CDMRP, W81XWH-16-1-0675.

${ }^{*}$ A.V.U. and P.F.K. contributed equally to this work.

${ }^{\dagger}$ D.K.C. and J.A.W. contributed equally to the support of this work.

Acknowledgments: We thank Mike Carman and Bill Pennie from the Research Instrumentation Shop at University of Pennsylvania (https://www.med.upenn.edu/ ris/index.html) for codeveloping a novel porcine stereotactic frame.

Correspondence should be addressed to John A. Wolf, PhD, Department of Neurosurgery, Perelman School of Medicine, 172A Stemmler Hall, 3450 Hamilton Walk, Philadelphia, PA, 19104. E-mail: wolfjo@pennmedicine.upenn.edu. DOI:http://dx.doi.org/10.1523/ENEURO.0102-18.2018

Copyright (C) 2018 Ulyanova, Koch et al.

This is an open-access article distributed under the terms of the Creative Commons Attribution 4.0 International license, which permits unrestricted use, distribution and reproduction in any medium provided that the original work is properly attributed. been done using both histologic and clinical imaging methods such as positron emission tomography and magnetic resonance imaging (Lind et al., 2007; Rogers et al., 2008). These methods identified features from both the rodent and the nonhuman primate hippocampus such as its position in the brain, a laminated hilus, and a dispersion of the deep pyramidal cells into stratum oriens similar to those of nonhuman primates (Holm and West, 1994; Lind et al., 2007; Sorensen et al., 2011). Recently, a comprehensive and detailed characterization of cortical surface anatomy and cytoarchitecture of the miniature pig's brain was done based on Nissl staining (Bjarkam et al., 2017). Here we histologically examine potential strain and age-related differences as well as the neuroanatomy of the miniature swine hippocampus in the sagittal and coronal plane.

Previous experimental and preclinical functional studies in both normal and pathophysiological states have been undertaken using functional MRI, electroencephalography, and most recently, both in vivo and ex vivo electrophysiology (Van Gompel et al., 2011; Wolf, 2017). As the value of the pig model in neurologic diseases becomes more evident, further characterization of the anatomy and connectivity of the porcine hippocampus is required. To perform in vivo electrophysiological studies using precisely placed high-density depth electrodes capable of recording laminar activity for comparative examination, we required a method of large animal stereotaxis without image guidance. We therefore implemented stereotaxis without the necessity of imaging to precisely place depth probes into the dorsal hippocampus of miniature pigs relative to the laminar structure of the CA1 region. We describe for the first time some of the basic characteristics of the electrophysiological laminar structure of the porcine hippocampus under anesthesia using custom high-density silicon laminar probes, including simultaneous recordings of single-unit activity and local field potentials (LFPs). We confirmed the laminar structure of the hippocampus and the precise location of the multichannel silicon probe using both electrophysiology and histopa- 
thology, reducing the need to immediately recover electrode tracks and increasing localization confidence during chronic implantations in future animals. Along with acute investigations, this methodology will ultimately allow for the potential expansion of hippocampal neurophysiology into awake behaving pigs, as well as in chronic models of pathophysiological conditions such as traumatic brain injury and epilepsy.

\section{Materials and Methods}

\section{Animals and animal care}

In the current study, we used a total of 17 male Yucatan miniature pigs, which are known for their docile temperament, ease of handling, and slow growth rate (NSRRC Cat\# 0012, RRID: NSRRC_0012). Two of them were used for histologic studies only, while 15 underwent both electrophysiological and histologic examinations. Pigs were purchased from Sinclair and underwent the current studies at the approximate age of 5-6 months at a mean weight of $37.94 \pm 3.26 \mathrm{~kg}(n=17$, mean \pm SEM). We chose this age as the pigs are post-adolescent with near-fully developed brains, but young enough to be of a manageable weight for procedures and behavior (Pampiglione, 1971; Flynn, 1984; Duhaime et al., 2000). All pigs were pair housed when possible, but always in a shared room with other pigs. All animal procedures were performed in accordance with the University of Pennsylvania animal care committee's regulations.

\section{Novel porcine stereotaxis}

Although stereotactic approaches based on skull landmarks, or on individual subject imaging, have been designed for in vivo electrophysiological studies in pigs, they are either inconsistent, expensive to use, or difficult to implement (Saito et al., 1998; Marcilloux et al., 1989; Félix, 1999). Our goal was to design a relatively inexpensive stereotactic system combining a freely available MRI atlas of pig anatomy (Saikali et al., 2010) with electrophysiological localization without the need for individual subject imaging to accomplish in vivo electrophysiological recordings across the laminar structure of the CA1 region of the pig hippocampus. Based on previous stereotactic work in our laboratory, we chose to select bregma as the starting landmark for our stereotaxis. Bregma is defined as an anatomic point on the skull at which the coronal sutures intersect with the sagittal suture at the midline (Fig. 2C). Although the relationship between bregma and the underlying brain can vary by several millimeters anterior to posterior (i.e., along the sagittal plane), the principle advantage is that bregma can be easily identified during surgery. We therefore defined the 2D medial-lateral (ML) and anterior-posterior (AP) zero point to be bregma. Based on our previous experience, we identified the approximate level of bregma in the coronal and sagittal planes on a freely available 3D MRI atlas of the pig brain (Saikali et al., 2010). Fig. 2A shows the orientation of the cortex and deeper structures, such as the dorsal and ventral hippocampus and the thalamus, at this level on the MRI atlas (also see Fig. 1B). Based on this MRI atlas, we then chose $7 \mathrm{~mm}$ lateral to the midline as the sagittal plane that maximized CA1 thickness and aligned the dor- sal hippocampal laminar structure perpendicularly with the cortical surface. Note that the Yorkshire (another pig strain) MRI atlas coronal section is structurally similar to a representative coronal section of the Yucatan brain used in this study, with slight shape variations due to strain differences (Figs. $1 B$ and $2 A$, see below).

Because the position of bregma in the AP plane with respect to the brain varies between animals, we selected an approximate range of AP coordinates that spans the AP extent of the dorsal hippocampus on the MRI atlas, $\sim 12 \mathrm{~mm}$ in total. A sagittal section through ML 7 taken from the MRI atlas shows the position and approximate size of the dorsal hippocampus (Fig. 2B, horizontal line, see below). The image is rotated so that cortical surface above the hippocampus is horizontal. Again, the sagittal section obtained from the MRI atlas is similar to the sagittal histologic section of porcine brain used in our study (Fig. $1 A$, bottom). This section of brain defined by the AP extent of the dorsal hippocampus at ML 7 then became the region of interest for electrophysiological mapping during surgery (see below).

\section{Placement in stereotactic frame}

We constructed a novel stereotactic frame based on percutaneously placed skull pin fixation, a commonly employed technique in human neurosurgery (Fig. 2C, see below). The stereotactic frame consisted of four major components: frame base, bite plate, anterior-posterior (AP) bars, and skull pins (Fig. 2C, see below). The frame base (1) hold all parts of the stereotaxis and was made of heavy aluminum alloy with holes to attach the AP bars of the stereotactic frame. As the animal was guided into the stereotactic frame, its jaw was opened and its maxilla placed on top of the bite bar (2) with the endotracheal tube positioned below the bar. The point at which the bite bar meets the frame base was adjustable. Additionally, the bite bar could be maneuvered in the AP plane and rotated in the coronal plane, allowing the researcher to optimally position the head before pin insertion. The AP bars (3) were independent parallel rails fastened to the frame base and run parallel to the animal's head in the sagittal plane. These arms had standard 1-mm markings. Stereotactic manipulator arms (Kopf Instruments, Cat\# 1760) could then be secured to the AP bars and moved along them in the AP plane using the Vernier scale to $0.1-\mathrm{mm}$ precision. Before securing the animal with the skull pins, two points on each side of the animal along the zygoma, $\sim 3 \mathrm{~cm}$ apart, were sterilely prepped with chlorhexidine solution and a small stab incision of 2-3 mm made at each point with a scalpel. Four stainless steel pins (4) affixed to the frame were inserted into the zygoma at each of these points, providing rigid fixation of the head to the frame. To position the head precisely for stereotactic localization, each of these pins could be moved in three dimensions while the pig was secured in the stereotaxis.

We used an iterative process of progressively finer adjustments both before and after skull pin fixation to place the pig's head precisely within the frame at a consistent angle such that the dorsal surface of the brain 

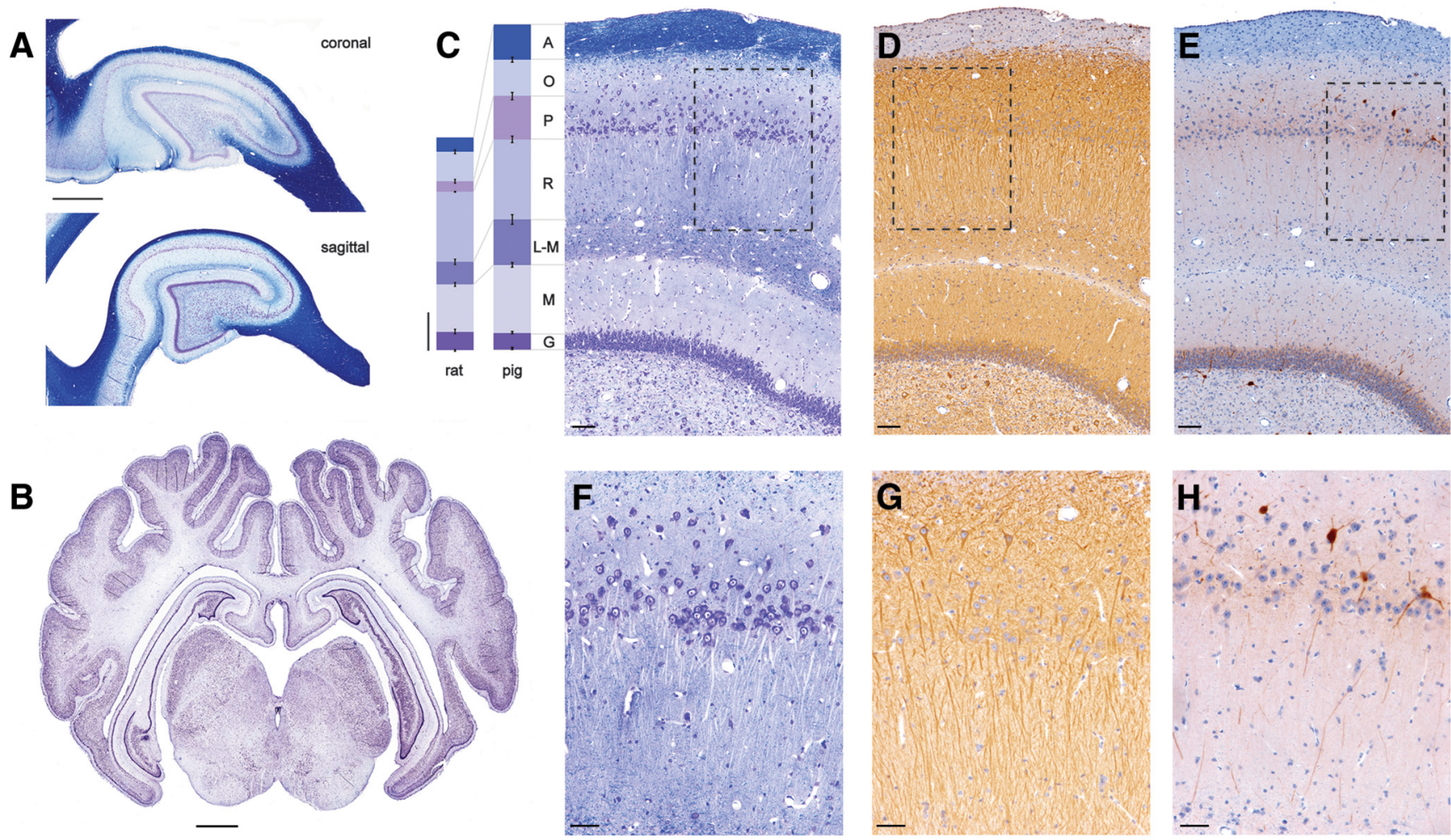

Figure 1. Morphology of porcine hippocampus. Sagittal and coronal sections of porcine brain were stained histologically to show organizational structure. A, Coronal (top) and sagittal (bottom) sections stained with LFB/CV show white and gray matter composition in dorsal hippocampus, which can be accessed stereologically from the brain surface. Both coronal and sagittal cuts through hippocampus produce arrow-like structures with consistent anatomic layers (scale $=2 \mathrm{~mm}$ ). $\boldsymbol{B}$, Coronal section stained with CV showing gyrencephalic structure (bregma $-1.5 \mathrm{~mm}$ ). Deep brain structures including hippocampus (dorsal and ventral) and thalamus are visible (scale bar $=2 \mathrm{~mm}$ ). $\boldsymbol{C}-\boldsymbol{E}$, Hippocampal layers including the alveus $(A)$, stratum oriens $(O)$, stratum pyramidale $(P)$, stratum radiatum $(R)$, stratum lacunosum-moleculare $(\mathrm{L}-\mathrm{M})$, stratum moleculare $(\mathrm{M})$, and stratum granulosum (G) are clearly visible (scale bar $=100 \mu \mathrm{m})$. C . Staining with LFB shows the myelinated axons, while counterstaining with CV highlights neurons in the pyramidal and dentate cell layers. Columns on the left show the width of corresponding hippocampal layers quantified from H\&E-stained sections in Yucatan pigs (bregma $-1.5 \mathrm{~mm} ; n=8$ ) and Long-Evans rats (bregma $-4.2 \mathrm{~mm} ; n=6$ ). The widths for the pig hippocampal layers are (in $\mu \mathrm{m}$, mean $\pm \mathrm{SEM}$ ) $\mathrm{A}=173.8 \pm 11.55, \mathrm{O}=178.3 \pm 15.01, \mathrm{P}=213.2 \pm 15.9, \mathrm{R}=394.1 \pm 22.36, \mathrm{~L}-\mathrm{M}=223.1 \pm 11.7, \mathrm{M}$ $=335.1 \pm 17.85, \mathrm{G}=81.3 \pm 4.5$. The corresponding widths for rat hippocampal layers are (in $\mu \mathrm{m}$ ): $\mathrm{A}=69.1 \pm 9.05, \mathrm{O}=144.4 \pm$ $7.51, \mathrm{P}=53.1 \pm 1.9, \mathrm{R}=343.9 \pm 12.33, \mathrm{~L}-\mathrm{M}=112.2 \pm 8.21, \mathrm{M}=232.8 \pm 11.35, \mathrm{G}=90.6 \pm 3.52$ (scale bar $=200 \mu \mathrm{m})$. The following hippocampal layers were significantly larger in pigs than in rats: stratum alveus by $152 \%(p<0.001)$, pyramidale by $301 \%$ $(p<0.001)$, radiatum by $15 \%(p<0.05)$, lacunosum-moleculare by $99 \%(p<0.001)$, and stratum moleculare by $44 \%(p<0.01)$, whereas the stratum oriens and granulosum were not significantly different. $\boldsymbol{D}$, Staining with MAP2 identifies location of dendritic arbors, which are densely packed. $\boldsymbol{E}, \mathrm{A}$ subpopulation of interneurons is identified using parvalbumin (PV) IHC in pyramidal and dentate granule cell layers. $\boldsymbol{F}-\boldsymbol{H}$, The pyramidal CA1 layer (scale bar $=50 \mu \mathrm{m}$ ). $\boldsymbol{F}$, The pyramidal CA1 cell layer is widely dispersed, similar to the human CA1 layer, as shown with LFB/CV staining. G. Pyramidal CA1 cells send their dendrites down toward the hippocampal fissure as highlighted with MAP2 staining. $\boldsymbol{H}, \mathrm{PV}^{+}$interneurons located above, inside, and below pyramidal CA1 layer send their projections into stratum radiatum.

above the hippocampus was parallel to the base of the stereotactic frame. The pig was first positioned approximately within the frame using a bite bar. The four skull pins were then inserted through the skin into the bone of bilateral zygomas, fixing the head. The head was then further adjusted to the desired angle using the skull pins, which can be moved in three dimensions. A final fine adjustment to the skull angle was made via direct measurements on the surface of the skull using a stereotactic arm and measurements on the frame once the skull had been exposed.

An angle of $10-12^{\circ}$ between the skin above the dorsal surface of the skull and the base of the stereotaxis was initially selected so that the cortical surface above the hippocampal region is level with the AP bars (Fig. 2B, dashed line, see below). Following initial alignment and skull fixation of the animal in the stereotactic frame to achieve this angle, the animal's head was repositioned again after skin incision based on fine measurements of the skull surface and bregma. An electrode holder with a marking pin was assembled and zeroed to bregma and the skull surface. To level the skull in the coronal plane, the dorsal-ventral (DV) coordinates were first measured in the ML plane. Head angle was finely adjusted if the difference in the DV coordinates on each side of the skull at $\mathrm{ML}=10 \mathrm{~mm}$ was $>0.5 \mathrm{~mm}$. Next, the DV coordinates 
A

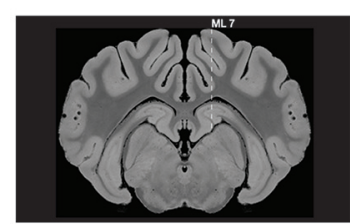

B

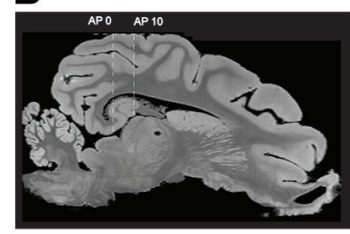

C

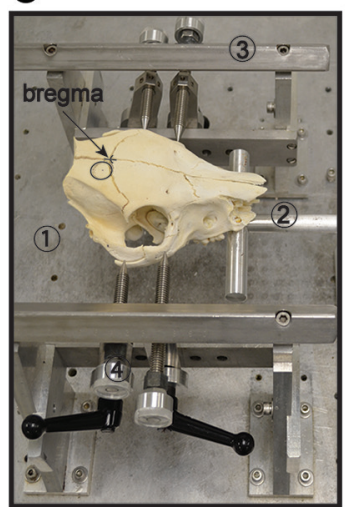

D

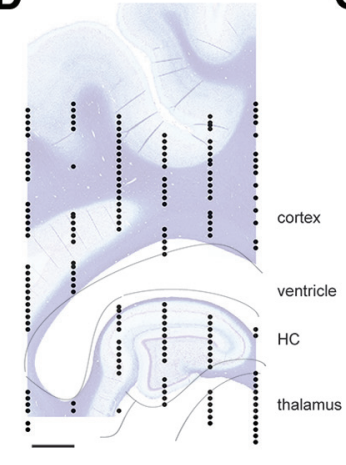

E

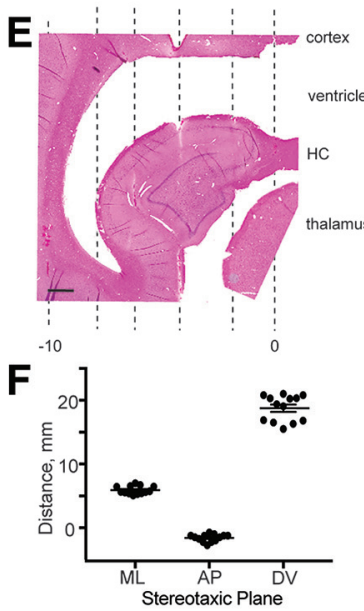

G

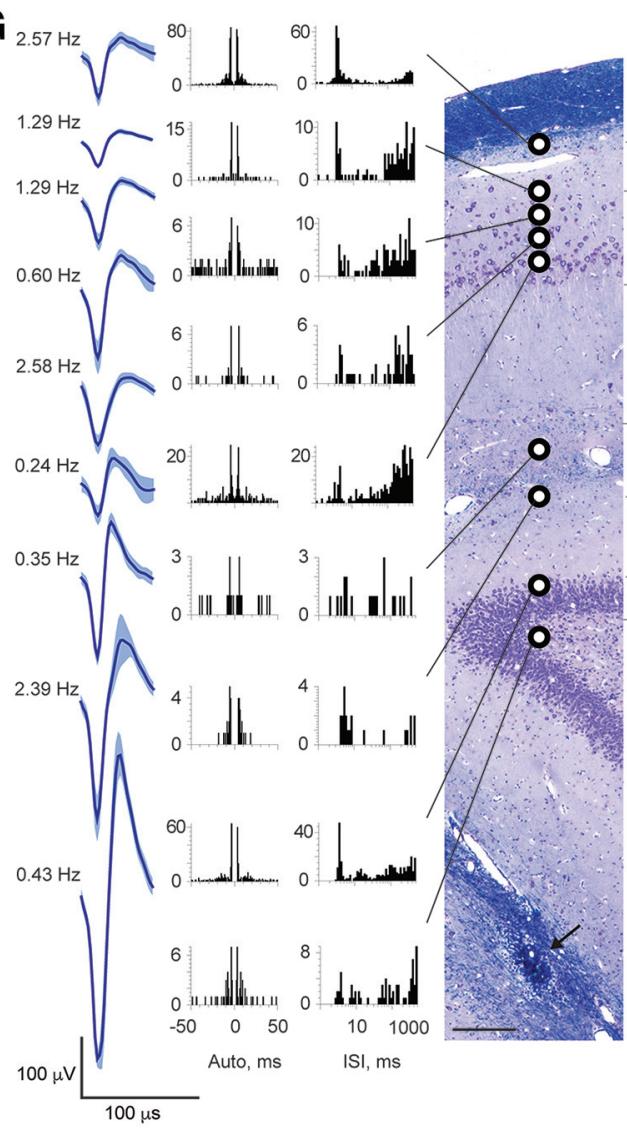

H

A

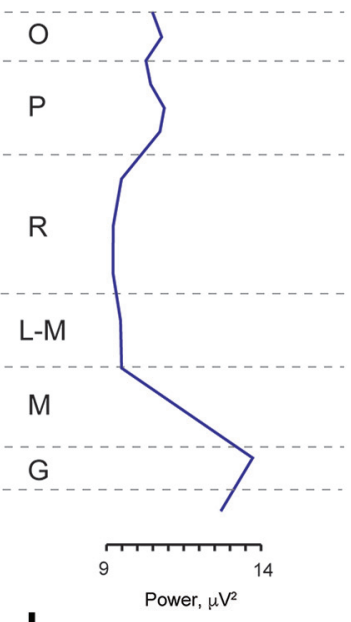

I

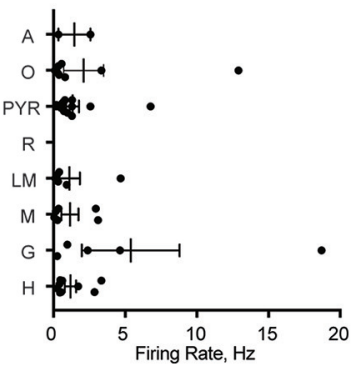

Figure 2. Single-unit recordings in porcine hippocampus corroborate laminar structure. $\boldsymbol{A}$, A representative coronal section from the MRI atlas (bregma $-1.5 \mathrm{~mm}$ ) within the 12-mm AP range of interest shows the orientation of deep structures such as dorsal and ventral hippocampi (Saikali et al., 2010). We selected ML 7 as an access plane to the widest part of dorsal hippocampus. $\boldsymbol{B}$, A sagittal section through ML 7 taken from the MRI atlas shows position and approximate size of the dorsal hippocampus. The image was rotated so that cortex above hippocampus is in the horizontal plane (horizontal dashed line). An approximate size of the hippocampus is shown between vertical dashed lines from right (AP 0) to left (AP -10). C, Photograph of a pig skull in situ in the novel stereotaxic instrument with an arrow pointing to bregma and a circle indicating a craniectomy site. The main parts of the stereotaxis are frame base (1), bite bar (2), anterior-posterior (AP) bars (3), and pins (4). The animal's head is first guided between AP bars and onto an adjustable bite bar, then a set of four pins is inserted into the zygomatic bones. Location and size of the craniectomy are shown (black circle). $\boldsymbol{D}, \mathrm{ML}$ coordinates obtained with this stereotaxis are fairly accurate, but AP coordinates are not. Therefore, electrophysiological mapping is needed for each experiment. The digitized tungsten map from our initial animal is shown in the AP plane at ML 7 overlying a representative sagittal section, stained with LFB/CV (scale bar = $2 \mathrm{~mm}$ ). The lines outline deep brain structures including subcortical white matter, ventricle, hippocampus, and thalamus. $\boldsymbol{E}$, A representative sagittal section from the initial animal stained with $\mathrm{H} \& \mathrm{E}$ (scale bar $=1 \mathrm{~mm}$ ) contains Hamilton syringe tracks in the AP plane in 2-mm steps from AP 0 to AP -10 (dashed lines). $\boldsymbol{F}$, The precision and reproducibility of our targeting technique in 13 animals was estimated histologically in the ML and AP planes and electrophysiologically in the DV plane. The estimated distances are (in $\mathrm{mm}$ ): $\mathrm{ML}=5.91 \pm 0.17$ from midline, $\mathrm{AP}=-1.60 \pm 0.16$ from bregma, and $\mathrm{DV}=18.76 \pm 0.57$ (mean $\pm \mathrm{SEM}$, $n=13$ ) from brain surface to first multiunit activity. G-I, Example of single spiking activity during the mapping procedure performed by slowly advancing a tungsten electrode along the indicated track. $\mathbf{G}$, A coronal section of the dorsal hippocampus stained with LFB/CV is shown from a subsequent animal from which tungsten electrode recordings were made during the initial mapping procedure (scale bar $=100 \mu \mathrm{m}$ ). Arrow points to the subsequently inserted silicon probe artifact. Histologically identified layers are labeled: alveus $(A)$, stratum oriens $(O)$, stratum pyramidale $(P)$, stratum radiatum $(R)$, stratum lacunosummoleculare (L-M), stratum moleculare $(M)$, stratum granulosum $(G)$. Three-minute recordings were made at regular intervals spaced 100-200 $\mu \mathrm{m}$ apart. Raw neural signals were processed offline, and putative single units were isolated. Depths at which single units were successfully isolated are indicated by black and white circles. Representative single units from each hippocampal layer are shown as averaged waveforms (mean \pm SD) accompanied by firing rates, autocorrelograms, and interspike interval histograms (Robertson et al., 2014). $\boldsymbol{H}$, To capture all multiunit activity and confirm the locations of the cellular layers, the RMS power of the high-frequency bandpass filtered signal $(600-6000 \mathrm{~Hz})$ was calculated. A representative profile of spiking activity shows several peaks at the corresponding pyramidal and granular cell layers. $I$, Firing rates of single units recorded in 2 animals with tungsten electrodes were averaged within each of 8 layers spanning $\sim 200 \mu \mathrm{m}$ each, corresponding to the hippocampal layers calculated in Fig. $1 \mathrm{C}$ (mean $\pm \mathrm{SEM}, n=2$ ). 
were measured in the midline at bregma and AP $+30 \mathrm{~mm}$. The angle between the skull surface and the base of stereotaxis was finely adjusted by repositioning the pins until the difference in the DV measurements at these two points was no more than $2.9 \pm 0.1 \mathrm{~mm}$, which corresponds to an angle of $7^{\circ}$. We found this angle to be ideal for reproducibly locating and mapping the dorsal hippocampus.

\section{Surgical procedure}

Yucatan miniature pigs were fasted for $16 \mathrm{~h}$ and induced with $20 \mathrm{mg} / \mathrm{kg}$ ketamine and $0.5 \mathrm{mg} / \mathrm{kg}$ midazolam. Animals were intubated with an endotracheal tube, and anesthesia was maintained with $2 \%-2.5 \%$ isoflurane per 2 liters $\mathrm{O}_{2}$. Each animal was placed on a ventilator and supplied oxygen at a tidal volume of $10 \mathrm{ml} / \mathrm{kg}$. A catheter was placed in an auricular vein to deliver $0.9 \%$ normal saline at $200 \mathrm{ml} / \mathrm{h}$. Additionally, heart rate, respiratory rate, arterial oxygen saturation, end tidal $\mathrm{CO}_{2}$, and rectal temperature were continuously monitored, while pain response to pinch was periodically assessed. All of these measures were used to titrate ventilation settings and isoflurane percentage to maintain an adequate level of anesthesia. A forced air warming system was used to maintain normothermia throughout the procedure.

Fifteen pigs in this study underwent electrophysiological recordings and analysis. These pigs were placed in a stereotactic frame (described above), with the surgical field prepped and draped. A linear incision was made along the midline. A 13-mm-diameter burr hole was made centered at $7 \mathrm{~mm}$ lateral to the midline and $4.5 \mathrm{~mm}$ posterior to bregma. The bony opening was subsequently expanded using Kerrison punches. The dura was opened in a cruciate manner using a \#11 blade.

\section{Electrophysiological mapping of porcine hippocampus}

In all 15 animals, the dorsal hippocampus was mapped with several tungsten electrode insertions in the sagittal plane, using observed spiking activity to generate a $2 \mathrm{D}$ map (Fig. 2D, see below). For this mapping, we used a high impedance tungsten monopolar electrode $\varnothing=125$ $\mu \mathrm{m}$, length $=60 \mathrm{~mm}$, impedance $=0.5 \mathrm{M} \Omega ; \mathrm{FHC}$, Cat\# UEWSEGSEBNNM). A skull screw was placed over the contralateral cortex as a reference signal.

We then chose coordinates within this map for insertion of either a custom-designed linear 32-channel silicon Edge probe with 200- $\mu \mathrm{m}$ spacing (NeuroNexus, Cat\# V1x32-Edge-10mm-200-312-Ref; $n=4$ ) or a customdesigned 32-channel silicon Vector probe (NeuroNexus, Cat\# V1 $\times 32-15 \mathrm{~mm}$-tet-lin-177; $n=11$ ) such that the spread of electrode contacts would span the laminar structure of the dorsal hippocampus at its maximal thickness in the DV plane, perpendicular to the individual hippocampal layers. The silicon probes were designed with one low-impedance channel placed $2 \mathrm{~mm}$ above the next most proximal channel, which we used as a reference signal (Fig. $3 A$, see below). For dorsal hippocampal targeting, this usually results in the reference channel being positioned within the temporal horn of the lateral ventricle sitting just above the hippocampus and provides
31 channels for intrahippocampal recordings. The custom-designed silicon Vector probe was similar to the custom-designed Edge linear silicon electrode, but the spatial arrangement of the 31 intrahippocampal channels were of a custom design (data not shown). At the end of the procedure, the anesthetic level was deepened (to 5\% isoflurane) and pigs were sacrificed for histologic analysis.

\section{Neural data collection and analysis}

All electrophysiological recordings were made under isoflurane anesthesia $(2 \%-2.5 \%)$. Neural signals were amplified and acquired continuously at $32 \mathrm{kHz}$ on a 64channel Digital Lynx 4SX acquisition system with Cheetah recording and acquisition software (Neuralynx).

\section{Spike detection and analysis}

Signals acquired from the tungsten monopolar electrode during the mapping procedure were bandpass filtered $(600$ $\mathrm{Hz}$ to $6 \mathrm{kHz}$ ) and thresholded for spike detection in real time. Thresholds were chosen based on observed signal-to-noise ratios during the session. Recorded spike trains and waveforms then underwent offline automated spike sorting using KlustaKwik software (http://klusta-team.github.io/klustakwik/, RRID: SCR_014480) and further manual refinements using SpikeSort3D (Neuralynx). Neural signals acquired from the 31 channels on the silicon probe were bandpass filtered $(0.1 \mathrm{~Hz}$ to $9 \mathrm{kHz})$ in real time before sampling. Offline spike detection and sorting was performed on the wideband signals using the Klusta package, which was developed for higher-density electrodes, and manually refined with KlustaViewa software (https://github.com/klusta-team/ klustaviewa). The Klusta routines are designed to take advantage of the spatial arrangement of and spike timing from all probe channels simultaneously in constructing putative clusters (Rossant et al., 2016). Resulting singleunit clusters were then imported into Matlab software (version R2017a, https://www.mathworks.com/products/ matlab.html, RRID: SCR_001622) for visualization and further analysis using custom and built-in routines. To minimize wave form shape distortion for visualization, the wideband signal was high-pass filtered using a wavelet multilevel decomposition and reconstruction filter (level 6, Daubechies 4 wavelet; Wiltschko et al., 2008). Waveforms were then extracted from this filtered signal and averaged for display. Spike trains were imported into NeuroExplorer software (version 6, http://www.neuroexplorer.com/, RRID:SCR_001818) for further analysis and to generate autocorrelograms and interspike interval histograms.

\section{Analysis of LFPs}

Acquired wideband LFPs recorded from 31 channels of the silicon probe were down-sampled to either 2 or $3 \mathrm{kHz}$ for further analysis. Signals were imported into Matlab software, version R2017a, and processed using a combination of custom and modified routines from the freely available Matlab packages FMAToolbox (http://fmatoolbox.sourceforge.net, RRID:SCR_015533), Chronux (http://chronux.org, RRID: SCR_005547), and EEGLAB (http://sccn.ucsd.edu/ eeglab/index.html, RRID:SCR_007292; Hazan et al., 2006; Mitra and Bokil, 2007). Current source density (CSD) was calculated as the second spatial derivative of the LFP across all 31 hippocampal channels and visualized by convention 

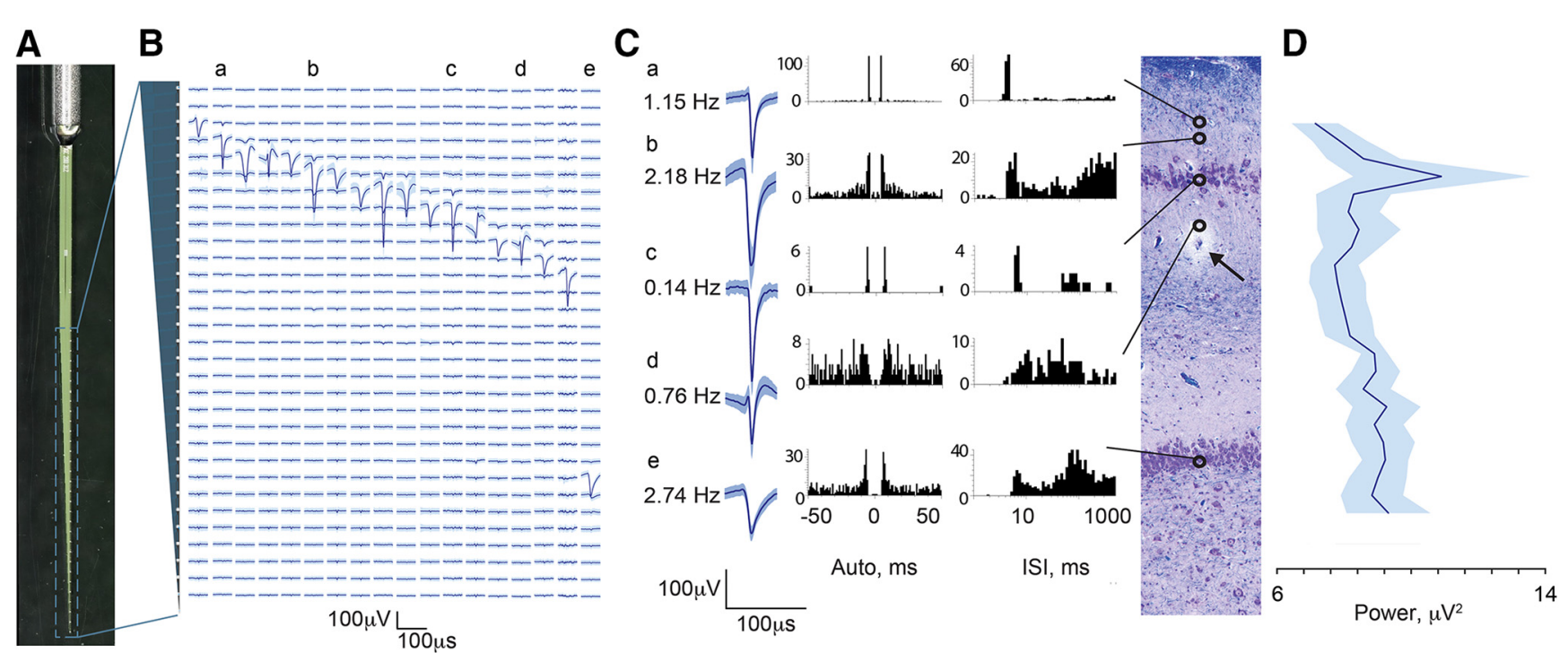

Figure 3. Single units recorded with silicon laminar probes confirm laminar structure. $\boldsymbol{A}$, Photograph of the custom-made Edge silicon probe shows an internal reference and linear orientation of its 31 channels. In each animal, this probe was inserted into the dorsal hippocampus based on initial electrophysiological mapping. Electrical activity $(\boldsymbol{B}-\boldsymbol{D})$ is shown as recorded from the final position of the probe. $\boldsymbol{B}$, Example in one animal of the laminar profile of single-unit activity shown as averaged waveforms (mean \pm SD) aligned to the 31 recording channels of the silicon probe along the vertical axis ( $200 \mu \mathrm{m}$ spacing), indicated by the gray cartoon of the probe on the left. Lowercase labels (a-e) indicate single-unit examples displayed in $\mathbf{C}$. $\mathbf{C}$, A coronal section of the dorsal hippocampus stained with LFB/CV is shown from the same animal (scale bar $=100 \mu \mathrm{m}$ ). Arrow points to silicon probe artifact, at approximately midshaft. Open circles indicate channels on which single spiking units were isolated revealing a large cluster in the region of stratum pyramidale and a single channel in the region of stratum granulosum (scale bar: $x$-axis, 2 ms; $y$-axis, $100 \mu \mathrm{V}$ ). Examples of isolated spike displayed as averaged waveforms (mean \pm SD) are shown along with autocorrelograms and interspike intervals histograms (Robertson et al., 2014). Alignment of single-unit examples to the histology was based on recovered artifact of the probe tip (not shown), stereotactic coordinates used during insertion, and known spacing of the 31 contacts. Vertical alignment is only approximate, as the plane of insertion was oblique to the histologic coronal plane and the relative thicknesses of the hippocampal layers change along the AP axis. $\boldsymbol{D}$, Stratum pyramidale of CA1 was identified in four animals recorded with the Edge laminar silicon probe based on the maximum power of multiunit spiking activity (RMS power in the $600-6000-\mathrm{Hz}$ band; mean \pm SEM, $n=4$ ). The traces from all four animals aligned at the peak of the RMS power are shown. Only aligned channels where all four animals overlapped are included (total channels displayed $=25$ out of 31 ).

as a heat map of current sinks (blue) and sources (orange; Mitzdorf, 1985) using the freely available Matlab package CSDplotter, version 0.1.1 (Pettersen et al., 2006).

To perform spectral power analysis across laminar structure, the hippocampal cellular layers were first identified in four animals recorded with the Edge laminar probe by calculating the root mean squared (RMS) power in the $600-6000-\mathrm{Hz}$ frequency band. The traces from multiple animals $(n=4)$ were then aligned at the peak of $600-6000-\mathrm{Hz}$ band, which corresponds to the pyramidal CA1 cell layer, and nonoverlapping channels were cut off (total channels displayed $=25$, out of $31, n=4$ ). After alignment, recordings from the 25 remaining hippocampal channels were broken into 1-s segments. Each segment underwent multitaper spectral analysis in the frequency ranges of theta $(4-10 \mathrm{~Hz})$, low gamma $(25-55 \mathrm{~Hz})$, high gamma $(55-90 \mathrm{~Hz})$, and a ripple frequency $(150-250 \mathrm{~Hz})$ range at each channel (Chernyy et al., 2008). The power for each 1-s segment at each channel was then integrated over the entire frequency range. The values for each channel were then averaged across all 1-s segments, resulting in the average integrated spectral power as a function of probe depth. For each animal, power spectral density (PSD) analysis of the channel presenting the maximum deflection during sharp-wave-like events was cal- culated in 1-20-Hz frequency band using Welch's method with the following parameters: window length $=1 \mathrm{~s}$, frequency resolution $=0.2 \mathrm{~Hz}$, overlap $=60 \%$. Each PSD was then normalized by its peak value and the average displayed as mean \pm SEM.

\section{Tissue handling and histologic examinations}

Histologic analyses were performed on brain tissue from male Yucatan miniature pigs ( $n=17$ total) to locate tracks generated by electrode insertion $(n=15)$ or to histologically characterize the porcine hippocampus $(n=$ 2). While under $5 \%$ isoflurane anesthesia, all animals underwent transcardial perfusion with $0.9 \%$ heparinized saline followed by $10 \%$ neutral buffered formalin (NBF). After postfixation for $7 \mathrm{~d}$ in $10 \% \mathrm{NBF}$ at $4^{\circ} \mathrm{C}$, each brain (weight $=88.29 \pm 2.12 \mathrm{~g}$, mean \pm SEM, $n=17$ ) was dissected into 5 -mm blocks in the coronal $(n=15)$ or sagittal $(n=2)$ planes and processed to paraffin using standard techniques. 8- $\mu \mathrm{m}$ sections were obtained at the level of the hippocampus in either the coronal or sagittal planes. Standard hematoxylin \& eosin (H\&E) staining was performed on all animals $(n=17)$ to visualize electrode tracks. Since electrode insertion could potentially disturb the cytoarchitecture, we used the hemisphere contralat- 
eral to the electrode insertion for histologic estimation of hippocampal layers.

\section{Luxol fast blue/cresyl violet (LFB/CV) staining}

Tissue sections were dewaxed in xylenes and rehydrated to water via graded ethanols before being immersed in $1 \%$ LFB solution (Sigma, S3382) at $60^{\circ} \mathrm{C}$ for 4 h. Excess stain was then removed by immersion of sections in $95 \%$ ethanol. Differentiation was performed via immersion in $0.035 \%$ lithium carbonate for $10 \mathrm{~s}$ followed by multiple immersions in $70 \%$ ethanol until the gray and white matter could be clearly distinguished. Slides were rinsed and counterstained via immersion in preheated $0.1 \%$ CV solution (Sigma, C5042) for $5 \mathrm{~min}$ at $60^{\circ} \mathrm{C}$. After further rinsing, slides were differentiated in $95 \%$ ethanol with $0.001 \%$ acetic acid, followed by dehydration, clearing in xylenes, and cover slipping using cytoseal-60.

Immunohistochemistry (IHC)

Single IHC labeling was performed according to previously published protocols. Briefly, tissue sections were dewaxed in xylenes, rehydrated to water via graded ethanols, and immersed in $3 \%$ aqueous hydrogen peroxide for 15 min to quench endogenous peroxidase activity. Antigen retrieval was achieved via microwave pressure cooker at high power for 8 min, submerged in Tris EDTA buffer ( $\mathrm{pH}$ 8.0). Sections were then incubated overnight at $4^{\circ} \mathrm{C}$ using antibodies specific for MAP2 (Abcam, Cat\# ab5392 Lot\# RRID: AB_2138153, 1:1000), parvalbumin (Millipore Cat\# MAB1572 Lot\# RRID: AB_2174013, 1:1000), and the N-terminal amino acids 66-81 of the amyloid precursor protein (APP; Millipore, Cat\# MAB348 Lot\# RRID: AB 94882, $1: 80 \mathrm{~K}$ ). Slides were then rinsed and incubated in the relevant species-specific biotinylated universal secondary antibody for $30 \mathrm{~min}$ at room temperature. Next, application of an avidin biotin complex (Vector Laboratories, Cat\# PK-6200 Lot\# RRID: AB_2336826) was performed for 30 $\mathrm{min}$, also at room temperature. Lastly, the 3,3'-diaminobenzidine (DAB) peroxidase substrate kit (Vector Laboratories, Cat\# SK-4100 Lot\# RRID: AB_233638) was applied according to manufacturer's instructions. All sections were counterstained with hematoxylin, dehydrated in graded ethanols, cleared in xylenes, and coverslipped using cytoseal-60.

\section{Slide imaging}

Whole-brain coronal tissue sections were digitally scanned at 20× magnification using the Aperio CS2 Digital Pathology Scanner (Leica Biosystems). Additional images were captured on a Nikon Eclipse 80i upright microscope and a DSRi1 camera (Nikon), with associated NIS-Elements software, version V4.5 (https://www.nikoninstruments.com/ Products/Software, RRID: SCR_014329).

\section{Comparative analysis of hippocampal architecture}

Comparative analysis of hippocampal layers between species was done on H\&E-stained sections. Hippocampal layers were measured on either scanned coronal sections of porcine brain $(n=8)$ or imaged coronal sections of archived formalin-fixed paraffin-embedded rat brain from male Long-Evans rats $(n=6)$. Whole-brain coronal sections at hippocampal level were stained with H\&E and imaged as described above. Individual hippocampal layers were visually identified and measured.

\section{Statistical analysis}

The data were analyzed using Graphpad Prism software, version 7 (http://www.graphpad.com/, PRID: SCR_002798). The histologic analysis of hippocampal cytoarchitecture in miniature Yucatan pigs $(n=8)$ and Long-Evans rats $(n=6)$ is presented as mean \pm SEM. For comparative analysis of hippocampal layers, corresponding depths of hippocampal layers in pigs were compared to those in rats ( $t$ test). The electrode placement and stereotactic precision are presented as mean \pm SEM $(n=13)$. Individual single-unit waveforms recorded with both tungsten electrodes and silicon laminar probes are presented as mean $\pm \mathrm{SD}$. Analysis of single-unit firing rates recorded with tungsten electrodes were averaged over $200 \mu \mathrm{m}$ distance and are presented as mean \pm SEM, $n=2$ pigs. Power spectrum analysis was performed in animals implanted with linear Edge probe and is presented as averaged power spectrum of various frequency bands (mean \pm SEM, $n=4$ ).

\section{Results}

\section{Neuroanatomy of porcine hippocampus}

To plan our initial stereotactic approach to the dorsal hippocampus in the Yucatan miniature pig, we first undertook a gross anatomic and histologic study of the structure. Three Yucatan miniature pigs were sacrificed and the brains extracted (see Methods). Fig. $1 B$ shows the sulci, multiple gyri, and white/gray matter composition of the porcine brain in coronal section (bregma $-1.5 \mathrm{~mm}$ ) stained with $\mathrm{CV}$, highlighting subcortical structures such as the hippocampus and thalamus that are stereotactically accessible from the brain surface. Analogous to rodent and human hippocampus, porcine hippocampus is oriented so that both coronal and sagittal planes produce arrow-like structures from anatomic layers in a similar orientation, as shown with LFB staining in Fig. $1 A$. The granule cells of the dentate gyrus form the point of the arrow, the notably large hilar region in pigs forms the body of the point, and the pyramidal cells of the cornu ammonis (CA) regions form the curved shaft in both planes. The classic hippocampal layers [alveus $(A)$, stratum oriens $(O)$, stratum pyramidale $(P)$, stratum radiatum $(R)$, stratum lacunosum-moleculare (L-M), stratum moleculare (M), and stratum granulosum (G)], as well as the hippocampal fissure, which divides the dentate from the CA regions, are clearly visible under higher magnification (Fig. 1C-E).

The width of each hippocampal layer was quantified from histologically stained sections of pig brain tissue used in this study (bregma $-1.5 \mathrm{~mm}$ ) and compared to archived, histologically stained sections of rat brain tissue at the similar hippocampal level (bregma $-4.20 \mathrm{~mm}$; see Fig. 1C). Columns show the widths of corresponding hippocampal layers quantified from H\&E-stained sections in pigs (mean \pm SEM, $n=8$ ) and rats (mean \pm SEM, $n=6$ ). The corresponding widths for pig hippocampal layers are (in $\mu \mathrm{m}$ ): $A=173.8 \pm$ $11.55, \mathrm{O}=178.3 \pm 15.01, \mathrm{P}=213.2 \pm 15.9, \mathrm{R}=394.1 \pm$ 22.36, $\mathrm{L}-\mathrm{M}=223.1 \pm 11.7, \mathrm{M}=335.1 \pm 17.85, \mathrm{G}=81.3$ \pm 4.5 . The corresponding widths for rat hippocampal layers 
are (in $\mu \mathrm{m}$ ): $\mathrm{A}=69.1 \pm 9.05, \mathrm{O}=144.4 \pm 7.52, \mathrm{P}=53.12$ $\pm 1.9, R=343.9 \pm 12.33, \mathrm{~L}-\mathrm{M}=112.2 \pm 8.21, \mathrm{M}=232.8$ $\pm 11.35, G=90.6 \pm 3.52$. The following hippocampal layers were significantly larger in pigs than in rats: stratum alveus by $152 \%(p<0.001)$, pyramidale by $301 \%(p<0.001)$, radiatum by $15 \%(p<0.05)$, lacunosum-moleculare by $99 \%$ $(p<0.001)$, and moleculare by $44 \%(p<0.01)$, whereas hippocampal layers stratum oriens and granulosum were not significantly different.

Staining with LFB/CV highlights myelinated fibers and neuronal cell bodies in the pyramidal and granular cell layers, revealing the expected ordered orientation of these fibers in relation to the cell bodies, a feature that plays critically in the laminar interpretation of hippocampal electrophysiology (Fig. 1C, F). The pyramidal CA1 cell layer appears less densely packed in pigs due to a progressive dispersion of these cells into stratum oriens in the direction of the subiculum as previously reported (Holm and West, 1994). This dispersion has the effect of partially blurring the distinction between stratum pyramidale and the most dorsal aspects of stratum radiatum, as synaptic inputs into the dendritic tree of the more dorsally located cells will colocalize with the more ventrally located cell bodies. This phenomenon can be readily seen with MAP2 labeling, showing pyramidal cells above the main CA1 layer sending dendrites through stratum pyramidale into the distal layers (Fig. 1D, G). These dendritic arbors are very dense and intermingled. Finally, as previously reported in another strain of pig, a subpopulation of interneurons located in the pyramidal and dentate granular cell layers can be identified with parvalbumin (PV) staining (Fig. 1E, H; Holm et al., 1990). These $\mathrm{PV}^{+}$interneurons primarily located above, inside, and below the pyramidal CA1 layer also have deep projections into the radiatum.

\section{Targeting the dorsal hippocampus using electrophysiological mapping}

We use electrophysiological mapping to address two different scales of localization: the localization of the probe within the 3D structure of the hippocampus, largely in the ML and AP planes, and the laminar localization of the probe with respect to the dendritic inputs along the DV axis of CA1. Since the initial, atlas-based coordinates selected for electrode placement can be inaccurate due to skull and individual animal variation as well as minor brain swelling during surgery, the precise location of the hippocampus needs to be determined within the stereotactic space. We used an electrophysiological mapping technique similar to that routinely employed clinically during deep brain stimulation implantation procedures (Gross et al., 2006), where a functional map of spiking activity in the cortex, dorsal hippocampus, and top of the thalamus was created by driving a small-diameter $(\varnothing=125 \mu \mathrm{m})$ high-impedance tungsten electrode into the brain and recording the coordinates at which neuronal spiking activity was detected.

We created a functional map of cortex and hippocampus by recording neuronal spiking activity at various depths, and we confirmed that electrophysiological activity of the dorsal hippocampus matches the anatomy using histologic needle artifacts from stereotaxic dye injections (Fig. 2). In our first experiment, mapping in our initial animal was executed in the sagittal plane $7 \mathrm{~mm}$ lateral to midline by advancing a single tungsten electrode in equal steps in the AP plane in 2-mm intervals (AP range: $0-10$ $\mathrm{mm})$. At each AP level, we drove the electrode from the brain surface $(D V=0)$ to a final depth of $30 \mathrm{~mm}(D V=30)$. We noted where multiunit spiking activity was detected along each track, thereby creating a 2D map of the dorsal hippocampus and surrounding structures in the sagittal plane (Fig. 2D, circles). The map of spiking activity generated an outline of the ventricle, dorsal hippocampus, thalamus, and cortex, shown overlying a representative sagittal section stained with LFB/CV (Fig. 2D). To assess whether the generated outline of electrophysiological activity corresponded to the histology, blue dye was stereotactically injected through a Hamilton syringe (26-gauge needle, outer diameter $\varnothing=0.46 \mathrm{~mm}$ ) along the same tracks as the tungsten electrode in the sagittal plane to a depth of $30 \mathrm{~mm}(n=2)$. After the animal was sacrificed, the brain was cut in the sagittal plane at the level of the hippocampus, revealing the blue dye tracks (not shown). We then cut serial sections at this level in the sagittal plane and stained them with H\&E, locating the track artifacts of the injection needle (Fig. 2E). We aligned the generated electrophysiological map with the sagittal section best showing the track artifacts (Fig. 2E, dashed lines), confirming accurate placement within the dorsal hippocampus. In subsequent experiments, the electrophysiological map generated was used to place a multichannel silicon probe within the dorsal hippocampus for subsequent experimental recordings (discussed below). In these animals, while electrophysiological mapping was still performed in the sagittal plane, brains were sectioned in the coronal plane, and sections demonstrating artifact from the silicon probe were located (Figs. 3 and 4, see below). After our initial experiments, this technique became routine, and we were able to use fewer tracks on later animals (as few as three) to localize the hippocampus in sagittal profile.

To assess the precision and reproducibility of our targeting technique within the 3D structure of the dorsal hippocampus, we estimated the final position of the inserted silicon probe histologically in the ML and AP planes and electrophysiologically in the DV plane (Fig. $2 F$, mean $\pm \mathrm{SEM}, n=13$ ). In the ML plane, the average measured histologic distance from the point of insertion into the dorsal hippocampus to the midline (in $\mathrm{mm}$ ) was $5.91 \pm$ 0.17 (mean \pm SEM, $n=13$ ). Compared to our stereotactic $\mathrm{ML}$ insertion point of $7 \mathrm{~mm}$ from midline, this is a discrepancy of $16 \%$, which is within the $11 \%-25 \%$ shrinkage range reported in the literature after formalin fixation (Quester and Schröder, 1997). In the AP plane, we estimated the position of the point of insertion into the dorsal hippocampus by comparing the histologic coronal section depicting the electrode tract with the MRI atlas (Saikali et al., 2010), choosing the coronal MRI slice $(0.25-\mathrm{mm}$ spacing) that best matched the overall hippocampal morphology in the histology. The average AP position of our probes in MRI space (in $\mathrm{mm}$ ) was $-1.60 \pm 0.16$ (mean \pm 


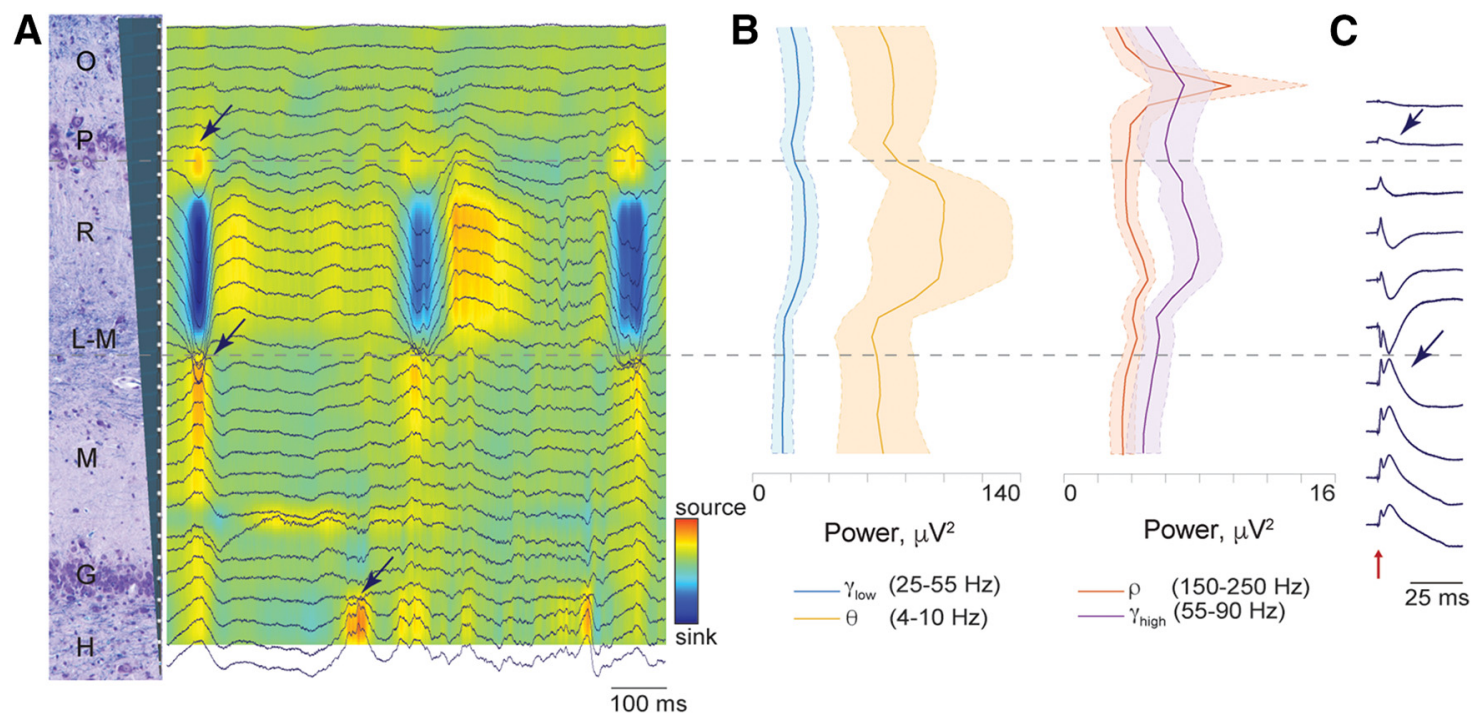

Figure 4. Laminar structure of extracellular recordings reveals electrode position. $\boldsymbol{A}$, Tracing of wide-band LFPs across all channels aligned to the 31 recording positions of the silicon Edge probe along the vertical axis (200- $\mu \mathrm{m}$ spacing) represented by the gray cartoon on the left. The signal was referenced to the skull ground screw. Multiunit spiking activity can be seen in a dorsal band indicating stratum pyramidale (approximately channel 4). Note regular bursts of synchronized activity that occur approximately every $300 \mathrm{~ms}$, potentially representing sharp wave-like activity under isoflurane anesthesia (Lustig et al., 2015). Arrows point to the phase reversal of the LFP above and below stratum radiatum as well as at dentate gyrus middle molecular layer. CSD map shows rhythmic large sink in stratum radiatum. B. Power spectra of classic theta (orange, 4-10 Hz), low gamma (blue, 25-55 Hz), high gamma (purple, $55-90 \mathrm{~Hz}$ ), and the ripple range (red, $150-250 \mathrm{~Hz}$ ) bands are shown aligned to the channels in $\boldsymbol{A}$. Each power band is presented as mean $\pm \operatorname{SEM}(n=4)$. Note that the theta band (orange) has maximal power in stratum radiatum. Although we found maximal power in the ripple band in stratum pyramidale (by definition the channel of maximal multiunit spiking activity; see Fig. 3D), we did not observe any definitive, discrete ripple oscillatory events. $\boldsymbol{C}$, Responses to perforant path stimulation (red arrow) were averaged over a 50 -ms time period (20 stimulations, $2 \mathrm{~s}$ apart, stimulation artifact removed for clarity). The laminar profile of these evoked potentials is shown in one animal. Phase reversals were observed above and below stratum radiatum (arrows).

SEM, $n=13)$. As a visual reference, the coronal MRI slice depicted in Fig. $2 A$ has an AP coordinate of -1.00 . Unlike localization in the ML and AP planes, our determination of DV precision was made by the electrophysiology. During insertion of the silicon probe, the most dorsal point within the dorsal hippocampus where neuronal spiking activity was detected, corresponding either to neurons within stratum oriens or to the pyramidal CA1 layer, was located an average of $18.76 \pm 0.57 \mathrm{~mm}$ (mean \pm SEM, $n=13$ ) below the brain surface in our stereotactic space.

\section{Hippocampal laminar structure and intra- hippocampal electrode localization in the DV axis}

Localizing within the AP and ML axes (in the sagittal and coronal planes, respectively) of the dorsal hippocampus as described above could also be accomplished with individual subject imaging and fiducial registration. However, localizing our probe precisely in the DV axis relative to the spiking activity in the CA1 pyramidal layer and dentate gyrus, as well as to the laminar structure of dendritic inputs between them, is more relevantly accomplished with electrophysiology, as neural activity is the direct measurement of interest. In two animals, once the electrophysiological map in the sagittal plane was created, we then recorded with the same high-impedance electrode at successive depths along a single track of our map to isolate and characterize the spiking activity as a function of depth. We advanced the electrode along this track in 100-200- $\mu \mathrm{m}$ intervals, recording for $3 \mathrm{~min}$ at each level. We then isolated putative single units offline. A larger multichannel silicon probe was then inserted to a depth of $25 \mathrm{~mm}$ along this same track as shown in Fig. $2 G$. The stereotactic coordinates of this probe as well as of the generated map, along with the histologic artifact generated by the silicon probe (Fig. 2G, black arrow), allowed us to approximately align the map of recorded single-unit activity with a representative coronal section from the same animal stained histologically with LFB/CV showing the probe artifact (coronal plane). This singleelectrode data revealed a cluster of single-unit activity aligned with the diffuse pyramidal cell layer depicted in the histology and a second cluster of single-unit activity aligned to the edge of the dentate gyrus at the granular cell layer and hilus. While various cell types in the hippocampus of the domestic pig have been identified with immunohistological markers previously, their electrophysiological characteristics are unknown (Holm et al., 1990, 1992, 1993). Here we report representative average waveforms (mean $\pm \mathrm{SD}$ ), autocorrelograms, and interspike interval histograms as well as firing rates of putative single-neuron action potentials within the various porcine hippocampal layers (Fig. 2G). The locations of the cell body layers in this animal were also confirmed by calculating multiunit spiking activity power in the $600-6000-\mathrm{Hz}$ frequency band (Fig. $2 H$ ). This demonstrates peaks in stratum pyramidale $(P)$ and stratum granulosum $(G)$. The firing rates of the individual single units recorded in both 
animals were then averaged in spatial bins of $\sim 200 \mu \mathrm{m}$ in depth (Fig. 2I), corresponding to the depths of the hippocampal layers calculated in Fig. $1 C$ (mean \pm SEM, $n=$ 2).

\section{Simultaneous laminar field potentials and single-unit activity via high-density silicon probe}

In a total of 15 animals, once the map of spiking activity of the dorsal hippocampus was created in the sagittal plane, we were able to place a multichannel silicon probe spanning the lamina of CA1 based on this map. In these experiments, we chose a point in the AP plane where we determined both that the CA1 layers were most perpendicular to the inserted probe and that showed the thickest span of spiking activity in the pyramidal layer along the DV axis in our electrophysiological map. We then inserted either the custom Vector or custom Edge 32 channel silicon probe (Edge probe depicted in Fig. $3 A$ : 31 recording sites, 200- $\mu \mathrm{m}$ spacing) into the dorsal hippocampus based on the initial mapping. We advanced the probe while monitoring spiking activity and the LFP on the multichannel probe in real time. In this way, we placed the large band of spiking activity corresponding to the pyramidal cell layer near the top of the probe (Fig. $3 B$ ). The internal reference on the silicon probe, located $2 \mathrm{~mm}$ above the first channel, is placed in the ventricle. Once in position, we recorded from the electrode for $3 \mathrm{~min}$.

We were then able to use multiple electrophysiological measurements and analyses of single- and multiunit spiking activity as well as LFPs to determine the location of the silicon probe relative to the laminar structure of the hippocampus. Figs. 3 and 4 illustrate these techniques. In these figures, the recorded 31 intrahippocampal channels (200- $\mu \mathrm{m}$ spacing) are, in turn, aligned to a coronal section of the dorsal hippocampus stained with LFB/CV from the same animal. Alignment of the probe relative to the histology was based on recovered artifact of the probe tips (not shown) and stereotactic coordinates used during insertion. Vertical alignment of histology and electrophysiology depicted in Figs. 2 and 3 are only approximate, as the plane of insertion was oblique to the histologic coronal plane and the relative thicknesses of the hippocampal layers change along the AP axis. In one illustrative animal, depicted in Fig. 3, isolation of single-unit spiking activity on all channels revealed a large cluster in the region of stratum pyramidale and a single channel in the region of stratum granulosum. In four animals, the position of the pyramidal cell layer was confirmed by calculating multiunit spiking activity power in the $600-6000 \mathrm{~Hz}$ frequency band (Fig. $3 D$, mean \pm SEM, $n=4$ ). The traces from these animals were aligned at the peak power of the 600-6000 $\mathrm{Hz}$ band, representing the pyramidal cell layer.

Several analyses of the LFPs also confirm precise localization of the probe within the hippocampus (Fig. 4). We first examined the wide-band LFPs across all 31 intrahippocampal channels (Fig. 4A). Inspection demonstrates prominent multiunit spiking activity in a band near the dorsal end of the electrode and a less prominent band of spiking activity more ventrally (Fig. $4 A$ ), which is also reflected in the isolated single-unit activity (Fig. $3 B, D)$. We also consistently observed waves of synchronized activity in stratum radiatum with a frequency of $2-3 \mathrm{~Hz}$ (Fig. 4A). These waves demonstrated a phase reversal at the pyramidal layer, at the bottom of stratum radiatum reminiscent of sharp waves described in the awake rodent hippocampus (Bragin et al., 1995b; Buzsáki et al., 2003), and again at dentate gyrus middle molecular layer (arrows). It is possible that these are regular sharp-wave-like (SPW-like) events representing synchronized presynaptic currents from CA3 projections, as SPWs have been shown to take on an oscillatory characteristic under isoflurane anesthesia in rodents (Lustig et al., 2015).

We also looked at the spectral power across the laminar structure of the porcine hippocampus in several frequency bands that are prominent in the rodent hippocampus (Bragin et al., 1995a), including classic theta $(\theta$, orange trace, 4-10 Hz), low gamma ( $\gamma_{\text {low }}$, blue trace, 25-55 Hz), high gamma ( $\gamma_{\text {high }}$, purple trace, 55-90 Hz), and ripple range ( $\rho$, red trace, $150-250 \mathrm{~Hz}$ ) bands (Fig. 4B, mean \pm SEM, $n=4)$. Oscillations within a classic theta frequency band (orange trace) appear prominently in stratum radiatum with a sharp fall-off toward the hippocampal fissure, whereas theta oscillations in the rodent demonstrate increasing power along the depth of CA1, peaking in stratum lacunosum-moleculare (Buzsáki et al., 2003) . This discrepancy could represent a difference in species or an effect of anesthesia. Low and high gamma bands (Fig. 4B, blue and purple traces) also have peaks of power in stratum radiatum as well as in stratum pyramidale. Since the traces from these four animals were aligned at the peak power of the $600-6000 \mathrm{~Hz}$ band (the pyramidal cell layer), 25 channels were left for the intrahippocampal laminar recordings and excluded the channels at the bottom of the probe positioned in the dentate gyrus layer (Fig. 4B, also see Fig. 3D).

Discrete oscillatory events within the frequency range of $150-250 \mathrm{~Hz}$ have been described in the pyramidal cell layer of the awake and asleep rodent hippocampus, termed "ripples" (Buzsáki, 2015). Although it is thought that ripple events in the LFP arise from a coordinated interaction between pyramidal cells and local interneurons, high-frequency oscillatory events in the pyramidal cell layer in general may have a significant component of their measured power derived from synchronized pyramidal cell action potentials. Importantly, much of the power may come from action potentials of cells further than 100 $\mu \mathrm{m}$ away, making this signal detectable even when there are no neurons close enough to a recording electrode to result in isolatable single-unit activity (Schomburg et al., 2012). This phenomenon may be particularly relevant in the current study, as we did not observe any definitive ripple oscillatory events in stratum pyramidale, most likely due to effects of anesthesia. Fig. $4 B$ shows integrated spectral power in this ripple band (150-250 Hz) across channels (red trace), demonstrating a large peak at the level of the prominent multiunit spiking activity, which is also the channel of maximum power within the 600-6000 $\mathrm{Hz}$ band (Fig. 3D), suggesting localization to the CA1 pyramidal layer. To further describe porcine hippocampal layers, we performed perforant path stimulation in one of 
the animals. The responses to the perforant path stimulation were averaged over a $50 \mathrm{~ms}$ time period (20 stimulations, $2 \mathrm{~s}$ apart). The laminar profile of the evoked potentials is shown in Fig. 4C. Two phase reversals were observed, above and below stratum radiatum hippocampal layer (Fig. 4C, arrows). Localized damage from the insertion of the electrode through the pyramidal CA1 cell layer was confirmed postmortem with LFB/CV staining (arrow, Fig. 3C). Additionally, these electrode tracks were confirmed with APP labeling, which accumulates in injured axons (Gentleman et al., 1993; Sherriff and Sivaloganathan, 1994; Johnson et al., 2013) and may be useful for electrode track detection in acute experiments in other species (data not shown).

\section{Discussion}

We describe here aspects of the laminar structure of the dorsal hippocampus of the Yucatan miniature pig both histologically and electrophysiologically using a methodology of stereotaxis that does not require individual subject imaging. This is important, as pigs have emerged as an attractive large animal model for neuroscience research and translational CNS disease models (Lind et al., 2007; Gieling et al., 2011b), yet little has been reported about the comparative laminar structure of the hippocampus and its electrophysiology. Due to their gyrencephalic brains and substantial white matter, pigs have been used to model CNS disorders such as TBI (Cullen et al., 2016) and epilepsy (Van Gompel et al., 2011) and may be useful for other hippocampal-centric models of cognitive dysfunction (Rollin, 2006; Maas et al., 2017). Further, these models are strengthened by the growing number of studies conducted to measure cognitive performance in pigs (Broom et al., 2009; Gieling et al., 2011a, b; Sullivan et al., 2013; Conrad and Johnson, 2015; Asher et al., 2016; Grimberg-Henrici et al., 2016; Schramke et al., 2016; Fleming and Dilger, 2017; Schuldenzucker et al., 2017).

Awake electrophysiological recordings in pigs have been reported in the past, and the use of pigs for intracranial monitoring studies and ex vivo electrophysiology has increased recently (Conn, 2017; Van Gompel et al., 2011; Wolf, 2017). One barrier to acute or chronic electrophysiology of the hippocampus has been the lack of methodology for targeting the hippocampus precisely without imaging guided stereotaxis. While several stereotaxic instruments have been developed for pigs, variations of skull landmarks due to age-dependent changes can create inconsistency in stereotaxic procedures (Szteyn et al., 1980; Poceta et al., 1981; Marcilloux et al., 1989; Saito et al., 1998; Ettrup et al., 2011). We have therefore developed an inexpensive, non-imaging-based swine stereotaxis for in vivo electrophysiology, which is based on skull position, angle, and landmarks and then refined with electrophysiological mapping. Localization within the dorsal hippocampus needs to be determined more precisely as well as independently for each subject when localization of layers is required for recordings or implantation (Saito et al., 1998). To accomplish this, we implemented electrophysiological mapping based on deep brain stimulation techniques widely used for neuro- surgical procedures (Gross et al., 2006). This allows us to target multichannel electrodes to the pyramidal CA1 cell layer and other hippocampal laminar structures during insertion and to reduce the reliance on histologic determination of suitable electrode placement within the hippocampus. While intra-operative recording to verify placement of chronic electrodes is not a standard procedure even in chronic primate electrophysiology, we suggest that it may complement existing image-guided techniques as more complex laminar probes begin to be used in large animals.

Our histologic investigation of the dorsal hippocampus of the Yucatan miniature pig confirms a preserved laminar structure across species with a standard orientation of the hippocampal layers. Our cytoarchitectural findings are consistent with a previously published, extensive immunohistological characterization of cell types in porcine hippocampus, including the progressive dispersion of pyramidal cells as one approaches the subiculum (Holm et al., 1990, 1992, 1993; Holm and West, 1994). Interestingly, we found that this variability in pyramidal cell density was reflected in the electrophysiology. Where the pyramidal cells were spread out, the peak power of the measured spiking activity was broad and less prominent than might be observed in the rodent (see Fig. 2H). Further, as seen in Fig. 1, this dispersion juxtaposes the pyramidal cell bodies of some cells with the dendritic arbors of others, potentially blurring the distinct layering of input and output currents (and thus the patterns of sinks and sources) classically seen in awake rodent electrophysiological studies. We also report the thickness of each hippocampal layer and directly compare them to those of the rat hippocampus. Porcine hippocampal layers including the alveus and strata pyramidale, radiatum, lacunosum-moleculare, and moleculare were significantly larger than those in rats, while other layers, namely strata oriens and granulosum, were not significantly different. This difference could reflect a disproportional increase in inputs from afferent cortex relative to local intrahippocampal circuitry as the gyrencephalic neocortex increases in size and complexity along the phylogenetic tree, while basic hippocampal function presumably remains largely preserved.

We have simultaneously recorded electrophysiological activity of many individual cells in the porcine dorsal hippocampus, which are appropriately distributed in a laminar fashion as expected from the histology and the LFP structure. The stereotaxic locations of the cell body layers were confirmed with a power analysis of the 600$6000 \mathrm{~Hz}$ frequency bands, which identifies multiunit spiking activity corresponding to strata pyramidale and granulosum. Importantly, our spectral power analysis of the ripple band $(150-250 \mathrm{~Hz}$ ) also identified the pyramidal cell layer (see Fig. 4B). As discussed above, power in this band may have significant contributions from synchronized pyramidal cell action potentials, including from cells more than $100 \mu \mathrm{m}$ from the recording electrode (Schomburg et al., 2012), a distance too great to routinely pick up spiking potentials. This could allow electrophysiological identification of the pyramidal layer in circumstances with- 
out detectable spiking activity, such as under deep anesthesia, or when only macro/low-impedance electrodes are used.

In addition to the ripple frequency band, we looked at the power of several other physiologically relevant bands expected in the hippocampus based on rodent experiments. Classic theta in the awake rodent hippocampus (4-10 Hz) shows a spectral power laminar pattern that increases from stratum radiatum to the hippocampal fissure, where it peaks, decreasing in deeper lamina (Bragin et al., 1995a). In contrast, we observed a broad peak in theta power in the stratum radiatum. Gamma oscillation power (25-100 Hz) in the awake rodent hippocampus shows a persistent increase from the stratum radiatum down to the hilus (Bragin et al., 1995a). Again, in contrast, we see only a broad peak in gamma power (25-55 Hz and 55-90 Hz) in stratum radiatum, with an additional peak in the pyramidal cell layer in the high gamma band. There could be a number of reasons for these discrepancies. First, the relative strengths and patterns of input into the pyramidal cell dendritic arbor may be fundamentally different in pigs compared to rats. This could result either in completely different laminar patterns or in a shift in the physiologically relevant frequency bands. An alternative or complementary explanation may be that the pattern and strength of inputs arriving into the dendritic arbor of the CA1 pyramidal cells are likely affected by the depth of anesthesia (Lustig et al., 2015).

There is also evidence that adequate depth of isoflurane anesthesia can drive the rodent hippocampus into a SPW-like state with bursts of inputs into stratum radiatum that demonstrate a pattern similar to CA3-derived SPW observed in the awake rodent (Lustig et al., 2015). We also observe regular bursts of synchronized input into stratum radiatum in the isoflurane-anesthetized pig that have the typical phase reversal across the pyramidal cell layer expected of CA3-derived SPWs. Consistent with the anesthetized state in the rodent (Lustig et al., 2015), we did not observe discrete ripple events, nor as prominent a ripple oscillation as those reported in behaving animals, but a peak in the ripple band frequency $(150-250 \mathrm{~Hz})$ in stratum pyramidale was present under anesthesia and correlates with the laminar profile of spiking activity (Buzsáki, 1989).

Our efforts to characterize network disruptions after TBI illustrate how the pig can also be used as a translational model of neurologic disorders, taking advantage of this characterization of the pig hippocampus and its electrophysiology. Electrophysiological changes have largely been studied in rodent models of TBI, including disruptions in long-term potentiation, synaptic plasticity, and broadband power (Miyazaki et al., 1992; Zhang et al., 2011; Paterno et al., 2015; Schmitt and Dichter, 2015; Villasana et al., 2015). In a pig TBI model, we have observed hippocampal axonal and synaptic dysfunction as well as regional hyperexcitability in an ex vivo slice preparation following an established porcine model of TBI where diffuse axonal injury is the primary neuropathological finding (Meaney et al., 1995; Cullen et al., 2016; Johnson et al., 2016; Wolf, 2017). Importantly, the elec- trophysiological characterization and methodology described here will permit the confirmation of these results in anesthetized and awake behaving swine using an in vivo electrophysiological procedure which utilizes laminar multielectrode silicon probes. This will further support investigations into hippocampal-centric translational models such as those being developed for the study of posttraumatic epilepsy, as well as laying the groundwork for closed-loop neuromodulation-based therapies in these models.

To summarize, we have characterized porcine laminar hippocampal electrophysiology under anesthesia using a new laminar probe and using techniques that do not require imaging-based stereotaxis. We have histologically confirmed that the neuroanatomy of Yucatan porcine brain closely resembles previously reported hippocampal structure, with features of both rodent and primate hippocampus. We have precisely placed depth probes into the dorsal hippocampus of miniature pigs and developed electrophysiology-based methodology to identify hippocampal layers and record hippocampal single units and LFPs simultaneously across these layers using multichannel probes. This will allow for analysis of spike-field interactions during normal and disease states. Laminar structure of hippocampus and the precise location of custom multichannel silicon probes were confirmed by electrophysiology and histopathology, reducing the future reliance on immediate recovery of electrode tracks. We hope that this characterization of the miniature swine hippocampus and stereotactic localization of the dorsal hippocampus will allow for greater use of these gyrencephalic mammals in translational models of hippocampal circuitry, as well as awake neurophysiology under healthy and pathophysiological conditions such as TBI and associated epilepsies. The combination of complex behaviors generated by these animals and the detailed neurophysiology of the hippocampus may present an ideal "middle ground" for translational hippocampal-centric disease models before clinical trials.

\section{References}

Asher L, Friel M, Griffin K, Collins LM (2016) Mood and personality interact to determine cognitive biases in pigs. Biol Lett 12: 20160402. CrossRef

Bjarkam CR, Glud AN, Orlowski D, Sørensen JCH, PalomeroGallagher N (2017) The telencephalon of the Göttingen minipig, cytoarchitecture and cortical surface anatomy. Brain Struct Function 222:2093-2114. CrossRef Medline

Bragin A, Jandó G, Nádasdy Z, Hetke J, Wise K, Buzsáki G (1995a) Gamma $(40-100 \mathrm{~Hz})$ oscillation in the hippocampus of the behaving rat. J Neurosci 15:47. Medline

Bragin A, Jando G, Nadasdy Z, van Landeghem M, Buzsaki G (1995b) Dentate EEG spikes and associated interneuronal population bursts in the hippocampal hilar region of the rat. $J$ Neurophysiol 73:1691. CrossRef

Broom DM, Sena H, Moynihan KL (2009) Pigs learn what a mirror image represents and use it to obtain information. Animal Behav 78:1037-1041. CrossRef

Buzsáki G (1989) Two-stage model of memory trace formation: a role for "noisy" brain states. Neuroscience 31:551-570. Medline

Buzsáki G (2015) Hippocampal sharp wave-ripple: a cognitive biomarker for episodic memory and planning. Hippocampus 25: 1073-1188. CrossRef Medline 
Buzsáki G, Buhl DL, Harris KD, Csicsvari J, Czéh B, Morozov A (2003) Hippocampal network patterns of activity in the mouse. Neuroscience 116:201-211. Medline

Chernyy N, Schiff SJ, Gluckman BJ, 2008. Multi-taper transfer function estimation for stimulation artifact removal from neural recordings. Conference proceedings: Annual International Conference of the IEEE Engineering in Medicine and Biology Society. IEEE Engineering in Medicine and Biology Society, p. 2772-2776.

Conn PM (2017) Animal models for the study of human disease. 2nd Edition. Cambridge, MA: Elsevier Science.

Conrad MS, Johnson RW (2015) The domestic piglet: an important model for investigating the neurodevelopmental consequences of early life insults. Ann Rev Animal Biosci 3:245-264. CrossRef

Cullen DK, Harris JP, Browne KD, Wolf JA, Duda JE, Meaney DF, Margulies SS, Smith DH, 2016. A porcine model of traumatic brain injury via head rotational acceleration. In: Kobeissy FH, Dixon CE, Hayes RL, Mondello S, editors. Injury Models of the Central Nervous System: Methods and Protocols. New York: Springer. $p$ 289-324.

Duhaime A-C, Margulies SS, Durham SR, O'Rourke MM, Golden JA, Marwaha S, Raghupathi R (2000) Maturation-dependent response of the piglet brain to scaled cortical impact. J Neurosurg 93:455462. CrossRef

Ettrup KS, Tornøe J, Sørensen JC, Bjarkam CR (2011) A surgical device for minimally invasive implantation of experimental deep brain stimulation leads in large research animals. J Neurosci Methods 200:41-46. CrossRef Medline

Félix BL, Albe-Fessard D, Marcilloux JC, Laplace JP (1999) Stereotaxic atlas of the pig brain. Brain Res Bull 49:1-137. Medline

Fleming SA, Dilger RN (2017) Young pigs exhibit differential exploratory behavior during novelty preference tasks in response to age, sex, and delay. Behavioural Brain Res 321:50-60. CrossRef Medline

Flynn T (1984) Developmental changes of myelin-related lipids in brain of miniature swine. Neurochem Res 9:935-45. Medline

Gentleman SM, Nash MJ, Sweeting CJ, Graham DI, Roberts GW (1993) $\beta$-Amyloid precursor protein ( $\beta$ APP) as a marker for axonal injury after head injury. Neurosci Lett 160:139-144. CrossRef

Gieling ET, Nordquist RE, van der Staay FJ (2011a) Assessing learning and memory in pigs. Animal Cogn 14:151-173. CrossRef Medline

Gieling ET, Schuurman T, Nordquist RE, van der Staay FJ, 2011b. The pig as a model animal for studying cognition and neurobehavioral disorders. In: Hagan JJ, editor. Molecular and Functional Models in Neuropsychiatry. Berlin: Springer. p 359-383.

Goodman S, Check E (2002) The great primate debate. Nature 417:684-687. CrossRef Medline

Grimberg-Henrici CGE, Vermaak P, Elizabeth Bolhuis J, Nordquist RE, van der Staay FJ (2016) Effects of environmental enrichment on cognitive performance of pigs in a spatial holeboard discrimination task. Animal Cogn 19:271-283. CrossRef

Gross RE, Krack P, Rodriguez-Oroz MC, Rezai AR, Benabid A-L (2006) Electrophysiological mapping for the implantation of deep brain stimulators for Parkinson's disease and tremor. Mov Disord 21:S259-S283. CrossRef

Hazan L, Zugaro M, Buzsáki G (2006) Klusters, NeuroScope, NDManager: a free software suite for neurophysiological data processing and visualization. J Neurosci Methods 155:207-216. CrossRef Medline

Holm IE, Geneser FA, Zimmer J (1992) Somatostatin- and neuropeptide $\mathrm{Y}$-like immunoreactivity in the dentate area, hippocampus, and subiculum of the domestic pig. J Comp Neur 322:390-408. CrossRef Medline

Holm IE, Geneser FA, Zimmer J (1993) Cholecystokinin-, enkephalin-, and substance P-like immunoreactivity in the dentate area, hippocampus, and subiculum of the domestic pig. J Comp Neur 331:310-325. CrossRef Medline

Holm IE, Geneser FA, Zimmer J, Baimbridge KG (1990) Immunocytochemical demonstration of the calcium-binding proteins calbindin-D 28k and parvalbumin in the subiculum, hippocampus and dentate area of the domestic pig. Progress Brain Res 83:8597.

Holm IE, West MJ (1994) Hippocampus of the domestic pig: a stereological study of subdivisional volumes and neuron numbers. Hippocampus 4:115-125. CrossRef Medline

Jang S-S, Chung HJ (2016) Emerging link between Alzheimer's disease and homeostatic synaptic plasticity. Neural Plast 2016: 7969272. CrossRef Medline

Johnson VE, Stewart W, Smith DH (2013) Axonal pathology in traumatic brain injury. Exp Neurol 246:35-43. CrossRef Medline

Johnson VE, Stewart W, Weber MT, Cullen DK, Siman R, Smith DH (2016) SNTF immunostaining reveals previously undetected axonal pathology in traumatic brain injury. Acta Neuropathol 131: 115-135. CrossRef Medline

Levin HS (2003) Neuroplasticity following non-penetrating traumatic brain injury. Brain Injury 17:665-674. CrossRef Medline

Lind NM, Moustgaard A, Jelsing J, Vajta G, Cumming P, Hansen AK (2007) The use of pigs in neuroscience: modeling brain disorders. Neurosci Biobehav Rev 31:728-751. CrossRef Medline

Lustig B, Wang Y, Pastalkova E (2015) Oscillatory patterns in hippocampus under light and deep isoflurane anesthesia closely mirror prominent brain states in awake animals. Hippocampus 26:102-109. CrossRef Medline

Maas AIR, Menon DK, Adelson PD, Andelic N, Bell MJ, Belli A, Bragge P, Brazinova A, Büki A, Chesnut RM, et al. (2017) Traumatic brain injury: integrated approaches to improve prevention, clinical care, and research. Lancet Neurol 16:987-1048. CrossRef

Marcilloux JC, Rampin O, Félix MB, Laplace JP, Albe-Fessard D (1989) A stereotaxic apparatus for the study of the central nervous structures in the pig. Brain Res Bull 22:591-597. CrossRef

Meaney DF, Smith DH, Shreiber DI, Bain AC, Miller RT, Ross DT, Gennarelli TA (1995) Biomechanical analysis of experimental diffuse axonal injury. J Neurotrauma 12:689-694. CrossRef Medline

Mitra PP, Bokil H, 2007. Observed brain dynamics. Oxford, UK: Oxford University Press, p. 408.

Mitzdorf U (1985) Current source-density method and application in cat cerebral cortex: investigation of evoked potentials and EEg phenomena. Physiol Rev 65:CrossRef

Miyazaki S, Katayama Y, Lyeth BG, Jenkins LW, DeWitt DS, Goldberg SJ, Newlon PG, Hayes RL (1992) Enduring suppression of hippocampal long-term potentiation following traumatic brain injury in rat. Brain Res 585:335-339. CrossRef

Pampiglione G (1971) Some aspects of development of cerebral function in mammals. Proc R Soc Med 64:429-435. Medline

Paterno R, Metheny H, Xiong G, Elkind J, Cohen AS (2015) Mild traumatic brain injury decreases broadband power in area CA1. J Neurotrauma 33:1645-1649. CrossRef

Pettersen KH, Devor A, Ulbert I, Dale AM, Einevoll GT (2006) Currentsource density estimation based on inversion of electrostatic forward solution: effects of finite extent of neuronal activity and conductivity discontinuities. J Neurosci Methods 154:116-133. CrossRef Medline

Poceta JS, Hamlin MN, Haack DW, Bohr DF (1981) Stereotaxic placement of cannulae in cerebral ventricles of the pig. Anat Record 200:349-356. CrossRef Medline

Quester R, Schröder R (1997) The shrinkage of the human brain stem during formalin fixation and embedding in paraffin. $J$ Neurosci Methods 75:81-89. Medline

Robertson CS, Hannay HJ, Yamal JM, Gopinath S, Goodman JC, Tilley BC, Epo Severe TBITI, Baldwin A, Rivera Lara L, SaucedoCrespo $\mathrm{H}$, et al. (2014) Effect of erythropoietin and transfusion threshold on neurological recovery after traumatic brain injury: a randomized clinical trial. JAMA 312:36-47. CrossRef

Rogers CS, Stoltz DA, Meyerholz DK, Ostedgaard LS, Rokhlina T, Taft PJ, Rogan MP, Pezzulo AA, Karp PH, Itani OA, et al. (2008) Disruption of the CFTR gene produces a model of cystic fibrosis in newborn pigs. Science 321:1837-1841. CrossRef Medline

Rollin BE (2006) The regulation of animal research and the emergence of animal ethics: a conceptual history. Theor Med Bioethics 27:285-304. CrossRef Medline 
Rossant C, Kadir SN, Goodman DFM, Schulman J, Hunter MLD, Saleem AB, Grosmark A, Belluscio M, Denfield GH, Ecker AS, et al. (2016) Spike sorting for large, dense electrode arrays. Nat Neurosci 19:634-641. CrossRef Medline

Saikali S, Meurice P, Sauleau P, Eliat P-A, Bellaud P, Randuineau G, Vérin M, Malbert C-H (2010) A three-dimensional digital segmented and deformable brain atlas of the domestic pig. J Neurosci Methods 192:102-109. CrossRef Medline

Saito T, Bjarkam CR, Nakamura M, Nemoto T (1998) Determination of stereotaxic coordinates for the hippocampus in the domestic pig. J Neurosci Methods 80:29-36. Medline

Schmitt S, Dichter MA, 2015. Electrophysiologic recordings in traumatic brain injury. In: Jordan G, Andres MS, editors. Handbook of Clinical Neurology. New York: Elsevier. p. 319-339.

Schomburg EW, Anastassiou CA, Buzsáki G, Koch C (2012) The spiking component of oscillatory extracellular potentials in the rat hippocampus. J Neurosci 32:11798. CrossRef Medline

Schramke S, Schuldenzucker V, Schubert R, Frank F, Wirsig M, Ott S, Motlik J, Fels M, Kemper N, Hölzner E, et al. (2016) Behavioral phenotyping of minipigs transgenic for the Huntington gene. $J$ Neurosci Methods 265:34-45. CrossRef

Schuldenzucker V, Schubert R, Muratori LM, Freisfeld F, Rieke L, Matheis T, Schramke S, Motlik J, Kemper N, Radespiel U, et al. (2017) Behavioral testing of minipigs transgenic for the Huntington gene-A three-year observational study. PLoS One 12:e0185970. CrossRef

Sherriff FEBL, Sivaloganathan S (1994) Early detection of axonal injury after human head trauma using immunocytochemistry for beta-amyloid precursor protein. Acta Neuropathol 87:55-62. CrossRef

Sorensen JC, Nielsen MS, Rosendal F, Deding D, Ettrup KS, Jensen KN, Jorgensen RL, Glud AN, Meier K, Fitting LM, et al. (2011)
Development of neuromodulation treatments in a large animal model: do neurosurgeons dream of electric pigs? Prog Brain Res 194:97-103.

Sullivan S, Friess SH, Ralston J, Smith C, Propert KJ, Rapp PE, Margulies SS (2013) Improved behavior, motor, and cognition assessments in neonatal piglets. J Neurotrauma 30:1770-1779. CrossRef Medline

Szteyn S, Galert D, Dynowski J, Hoczyk W (1980) The stereotaxic configuration of hypothalamus nerve centres in the pig. Anatomischer Anzeiger 147:12-32. Medline

Van Gompel JJ, Bower MR, Worrell GA, Stead M, Meier TR, Goerss SJ, Chang S-Y, Kim I, Meyer FB, Marsh WR, et al. (2011) Swine model for translational research of invasive intracranial monitoring. Epilepsia 52:e49-e53. CrossRef

Villasana LE, Kim KN, Westbrook GL, Schnell E (2015) Functional integration of adult-born hippocampal neurons after traumatic brain injury. eNeuro 2:0056-15.2015. CrossRef

Vink R (2018) Large animal models of traumatic brain injury. J Neurosci Research 96:527-535. CrossRef Medline

Wiltschko AB, Gage GJ, Berke JD (2008) Wavelet filtering before spike detection preserves waveform shape and enhances singleunit discrimination. J Neurosci Methods 173:34-40. CrossRef Medline

Wolf JA, Johnson BN, Johnson VE, Putt ME, Browne KD, Mietus CJ, Brown DP, Wofford KL, Smith DH, Grady MS, Cohen AS, Cullen DK (2017) Concussion induces hippocampal circuitry disruption in swine. J Neurotrauma 34:2303-2314. CrossRef

Zhang B, Chen X, Lin Y, Tan T, Yang Z, Dayao C, Liu L, Jiang R, Zhang J (2011) Impairment of synaptic plasticity in hippocampus is exacerbated by methylprednisolone in a rat model of traumatic brain injury. Brain Res 1382:165-172. CrossRef 